\title{
Entanglement of two harmonic modes coupled by angular momentum
}

\author{
L. Rebón and R. Rossignoli \\ Departamento de Física-Instituto de Física de La Plata, Universidad Nacional de La Plata, \\ C.C. 67, 1900 La Plata, Argentina
}

(Received 18 September 2011; published 18 November 2011)

\begin{abstract}
We examine the entanglement induced by an angular momentum coupling between two harmonic systems. The Hamiltonian corresponds to that of a charged particle in a uniform magnetic field in an anisotropic quadratic potential or, equivalently, to that of a particle in a rotating quadratic potential. We analyze both the vacuum and thermal entanglement, thereby obtaining analytic expressions for the entanglement entropy and negativity through the Gaussian state formalism. It is shown that vacuum entanglement diverges at the edges of the dynamically stable sectors, increasing with the angular momentum and saturating for strong fields, whereas at finite temperature entanglement is nonzero just within a finite field or frequency window and no longer diverges. Moreover, the limit temperature for entanglement is finite in the whole stable domain. The thermal behavior of the Gaussian quantum discord and its difference from the negativity is also discussed.
\end{abstract}

DOI: $10.1103 /$ PhysRevA.84.052320

PACS number(s): 03.67.Mn, 03.65.Ud, 05.30.Jp

\section{INTRODUCTION}

The investigation of quantum entanglement and quantum correlations in distinct physical systems is of great interest for both quantum information and many-body physics [1-4]. While the evaluation of entanglement in systems with a highdimensional Hilbert space is generally a difficult problem, boson systems described by quadratic Hamiltonians in the basic boson operators offer the invaluable advantage of admitting an exact evaluation of entanglement measures in both the ground and thermal states through the Gaussian state formalism [5-12]. The latter allows the entanglement entropy [13] and negativity $[14,15]$ of any bipartition of a Gaussian state to be expressed in terms of the symplectic eigenvalues of covariance matrices of the basic operators. Moreover, the positive partial transpose separability criterion $[16,17]$ is both necessary and sufficient for two-mode mixed Gaussian states [5] [as well as $(1, n-1)$ bipartitions of $n$-mode Gaussian states [7]], turning the negativity into a rigorous entanglement indicator for these systems. There is presently a great interest in continuous-variable-based quantum information [18] where Gaussian states constitute the basic element.

In addition, an approximate yet analytic evaluation of the quantum discord $[19,20]$ in two-mode Gaussian states was recently achieved $[21,22]$ by restricting the local measurement that determines this quantity to a Gaussian measurement [18]. Quantum discord is a measure of quantum correlations that coincides with the entanglement entropy in pure states but differs essentially from entanglement in mixed states, where it can be nonzero even if the state is separable, i.e., with no entanglement. The current interest in quantum discord was triggered by its presence [23] in certain mixed-statebased quantum computation schemes that provide exponential speedup over classical ones [24] yet exhibit no entanglement [25]. Important properties of states with nonzero discord were recently unveiled [26-28].

The aim of this work is to examine, using the Gaussian state formalism, the entanglement and quantum correlations between two harmonic modes generated by an angular momentum coupling. Such a system arises, for instance, when considering a charged particle in a uniform magnetic field in an anisotropic quadratic potential or a particle in a rotating anisotropic harmonic trap [29-32]. The model has been employed in several areas, including the description of deformed rotating nuclei [31,32], anisotropic quantum dots in a magnetic field [33], and fast rotating Bose-Einstein condensates [34-37] in the lowest Landau level approximation [38-40]. Containing just quadratic couplings in the associated boson operators, the different terms in the Hamiltonian may also be simulated in principle by standard optical means $[18,41]$. For a general quadratic potential, the model exhibits a complex dynamical phase diagram [42], presenting distinct types of stable and unstable domains and admitting the possibility of stabilizing an initially unstable system by increasing the field or frequency [42]. The model provides an interesting and physically relevant scenario for analyzing the behavior of mode entanglement in different regimes and near the onset of different types of instabilities, with the advantage of allowing an exact analytic evaluation of entanglement and quantum correlation measures at both zero and finite temperatures. In addition, the present results indicate that mode entanglement can be easily controlled in this system by modifying the field or frequency, thus suggesting a potential for quantum information applications. The dynamics of entanglement in other two-mode systems was examined in Refs. [43-45].

In Sec. II we describe the model and derive the analytic expressions for the vacuum entanglement entropy and the thermal negativity. The basic features of quantum discord are also discussed. The detailed behavior of entanglement with the relevant control parameters is then analyzed in Sec. III, where we show that while vacuum entanglement diverges at the edges of stable sectors, being correlated with the angular momentum, at finite temperature entanglement is finite and nonzero just within a finite-field window and below a finite limit temperature. A comparison between the thermal behavior of the negativity and that of the Gaussian quantum discord is also made, which indicates a quite different thermal response of these two quantities, with the discord vanishing only asymptotically for $T \rightarrow \infty$. We summarize in Sec. IV. 


\section{FORMALISM}

\section{A. Model Hamiltonian}

We consider a system described by the Hamiltonian

$$
H=\frac{1}{2}\left(P_{x}^{2}+k_{x}^{\prime} Q_{x}^{2}\right)+\frac{1}{2}\left(P_{y}^{2}+k_{y}^{\prime} Q_{y}^{2}\right)-\omega\left(Q_{x} P_{y}-Q_{y} P_{x}\right),
$$

which represents two harmonic modes coupled by an angular momentum term. Here $Q_{\mu}$ and $P_{\mu}$ stand for dimensionless coordinates and momenta $\left(\left[Q_{\mu}, P_{\nu}\right]=i \delta_{\mu \nu}\right.$ and $\left[Q_{\mu}, Q_{\nu}\right]=$ $\left.\left[P_{\mu}, P_{\nu}\right]=0\right)$. Equation (1) arises, for instance, in the description of a particle of charge $e$ and mass $m$ in a general quadratic potential subject to a uniform magnetic field, parallel to a principal axis of the potential. Denoting this axis as $z$, such a Hamiltonian reads

$$
\begin{aligned}
\mathcal{H} & =\frac{(\boldsymbol{P}-e \boldsymbol{A} / c)^{2}}{2 m}+\frac{1}{2}\left(K_{x} \mathcal{Q}_{x}^{2}+K_{y} \mathcal{Q}_{y}^{2}+K_{z} \mathcal{Q}_{z}^{2}\right) \\
& =\frac{1}{2}\left[\frac{\mathcal{P}_{x}^{2}+\mathcal{P}_{y}^{2}}{m}+K_{x}^{\prime} \mathcal{Q}_{x}^{2}+K_{y}^{\prime} \mathcal{Q}_{y}^{2}-\Omega \mathcal{L}_{z}\right]+\mathcal{H}_{z},
\end{aligned}
$$

where $\mathbf{A}=\frac{1}{2} \boldsymbol{H} \times \boldsymbol{Q}$ is the vector potential, $\Omega=\frac{e|\mathbf{H}|}{m c}$ is the cyclotron frequency, $\mathcal{L}_{z}=\mathcal{Q}_{x} \mathcal{P}_{y}-\mathcal{Q}_{y} \mathcal{P}_{x}, \mathcal{H}_{z}=\frac{1}{2}\left(\frac{\mathcal{P}_{z}^{2}}{m}+\right.$ $K_{z} \mathcal{Q}_{z}^{2}$ ), and $K_{\mu}^{\prime}=K_{\mu}+m \Omega^{2} / 4$. Equation (3) is also identical to the intrinsic Hamiltonian of a particle of mass $m$ in a quadratic potential of constants $K_{\mu}^{\prime}$ rotating around the $z$ axis with frequency $\Omega / 2$ [31,32].

The replacements $\mathcal{P}_{\mu}=P_{\mu} \sqrt{\hbar m \Omega_{0}}$ and $\mathcal{Q}_{\mu}=$ $Q_{\mu} / \sqrt{m \Omega_{0} / \hbar}$ in Eq. (3), with $\Omega_{0}$ a reference frequency, leads to $\mathcal{H}=\hbar \Omega_{0} H+\mathcal{H}_{z}$, with $H$ given by Eq. (1) and

$$
k_{\mu}^{\prime}=k_{\mu}+\omega^{2}, \quad k_{\mu}=\frac{K_{\mu}}{m \Omega_{0}^{2}}, \quad \omega=\frac{\Omega}{2 \Omega_{0}} .
$$

We note that in terms of the boson operators $b_{\mu}=\left(Q_{\mu}+\right.$ $\left.i P_{\mu}\right) / \sqrt{2}$, the scaled angular momentum $L_{z}=\mathcal{L}_{z} / \hbar$ in Eq. (1) is

$$
L_{z}=Q_{x} P_{y}-Q_{y} P_{x}=-i\left(b_{x}^{\dagger} b_{y}-b_{y}^{\dagger} b_{x}\right),
$$

which can then be simulated by standard linear optics, although for such bosons the first two terms in Eq. (1) become $\sum_{\mu=x, y} g_{\mu}^{+}\left(b_{\mu}^{\dagger} b_{\mu}+\frac{1}{2}\right)+g_{\mu}^{-}\left(b_{\mu}^{2}+b_{\mu}^{\dagger}{ }^{2}\right)$, with $g_{\mu}^{ \pm}=\frac{k_{\mu}^{\prime} \pm 1}{2}$, and require nonlinear means. If $K_{x}^{\prime}>0$, we can set $k_{x}^{\prime}=1$, i.e., $g_{x}^{-}=0$, by adequately choosing $\Omega_{0}$ in Eq. (4), but $g_{y}^{-}$ remains nonzero in the relevant anisotropic case $k_{y}^{\prime} \neq k_{x}^{\prime}$, where $\left[H, L_{z}\right] \neq 0$. The change to normal $x$ and $y$ bosons, such that $H=\sum_{\mu=x, y} \sqrt{k_{\mu}^{\prime}}\left(\tilde{b}_{\mu}^{\dagger} \tilde{b}_{\mu}+\frac{1}{2}\right)-\omega L_{z}$, will lead instead to an additional term proportional to $\left(1-\sqrt{k_{x}^{\prime} / k_{y}^{\prime}}\right)\left(\tilde{b}_{x} \tilde{b}_{y}-\tilde{b}_{x}^{\dagger} \tilde{b}_{y}^{\dagger}\right)$ in $L_{z}$.

\section{B. Diagonalization and stability}

If the parameter

$$
\Delta=\sqrt{\left(k_{x}^{\prime}-k_{y}^{\prime}\right)^{2} / 4+2 \omega^{2}\left(k_{x}^{\prime}+k_{y}^{\prime}\right)}
$$

is nonzero, the canonical transformation

$$
P_{\mu}^{\prime}=P_{\mu}+\gamma Q_{-\mu}, \quad Q_{\mu}^{\prime}=\frac{Q_{\mu}-\eta P_{-\mu}}{1+\eta \gamma},
$$

where $\gamma=\frac{2 \Delta-k_{x}^{\prime}+k_{y}^{\prime}}{4 \omega}, \eta=\frac{2 \gamma}{k_{x}^{\prime}+k_{y}^{\prime}}$, and the labels $(x, y)$ are now identified with $(+,-)$, allows Eq. (1) to be written as [42]

$$
H=\sum_{\mu= \pm} \frac{1}{2}\left(\alpha_{\mu} P_{\mu}^{\prime 2}+\beta_{\mu} Q_{\mu}^{\prime 2}\right)
$$

where $\alpha_{ \pm}=1-\frac{\omega}{\Delta}(\gamma \mp \omega)$ and $\beta_{ \pm}=\frac{\Delta}{\omega}(\gamma \pm \omega)$. If $\Delta=0$ and $\omega \neq 0$, a separable representation of the form of Eq. (8) in terms of canonical variables $\left(\left[Q_{\mu}^{\prime}, P_{\nu}^{\prime}\right]=\delta_{\mu \nu}\right.$ and $\left[P_{\mu}^{\prime}, P_{\nu}^{\prime}\right]=\left[Q_{\mu}^{\prime}, Q_{\nu}^{\prime}\right]=0$ ) is not feasible. Such a possibility can arise in the repulsive case $k_{\mu} \leqslant 0$, when the $4 \times 4$ matrix representing the quadratic form in Eq. (1) is not diagonalizable with the symplectic metric and leads to nontrivial Jordan forms [42].

For general real values of $k_{\mu}^{\prime}$ in Eq. (1), the coefficients $\alpha_{\mu}$ and $\beta_{\mu}$ in Eq. (8) can be positive, zero, negative, and even complex [42]. We will consider here those cases for which Eq. (8) can be further written as

$$
H=\sum_{\mu= \pm} \lambda_{\mu}\left(b_{\mu}^{\prime \dagger} b_{\mu}^{\prime}+\frac{1}{2}\right),
$$
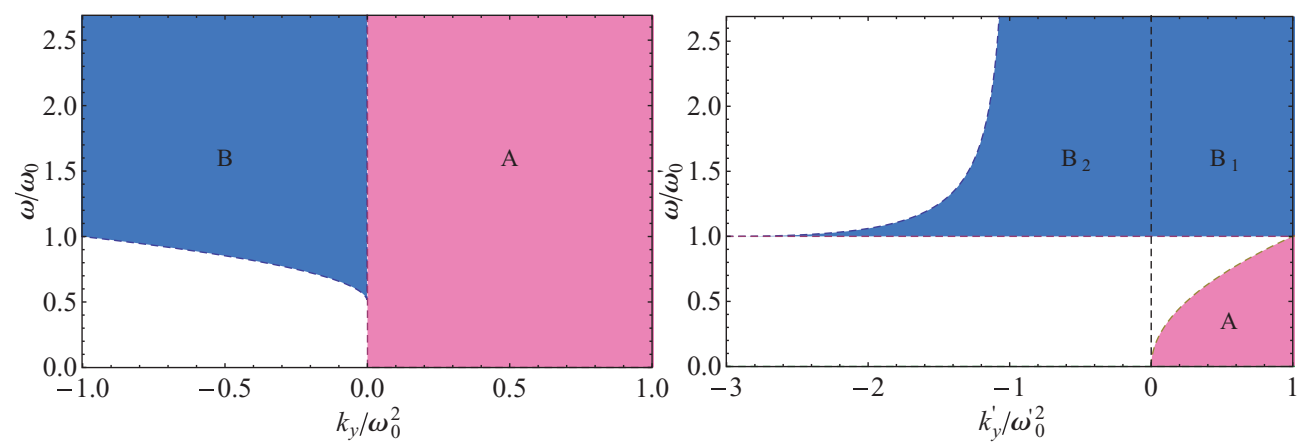

FIG. 1. (Color online) Left: Sectors with a discrete spectrum at fixed $k_{\mu}$ (particle in a magnetic field plus quadratic potential) in the anisotropy-frequency plane $\left(k_{y} /\left|k_{x}\right|, \omega / \omega_{0}\right)$, where $\omega_{0}=\sqrt{\left|k_{x}\right|}$. The positive-definite sector $A$ corresponds to an attractive potential $k_{\mu}>0$ for $\mu=x, y$, whereas the nonpositive sector $B$ corresponds to a repulsive potential $k_{\mu}<0$ for $\mu=x, y$ with $|\omega|>\omega_{c}$ [Eq. (11)]. Right: Same sectors at fixed $k_{\mu}^{\prime}>0$ (particle in a rotating quadratic potential) in the $\left(k_{y}^{\prime} / k_{x}^{\prime}, \omega / \omega_{0}^{\prime}\right)$ plane, where $\omega_{0}^{\prime}=\sqrt{k_{x}^{\prime}}$ and we have set $k_{x}^{\prime}>0$. Here $A$ is the positive-definite sector [Eq. (12)], $B_{1}$ is the nonpositive sector with $k_{y}^{\prime}>0$ [Eq. (13)], and $B_{2}$ is the nonpositive sector with $k_{y}^{\prime}<0$ [Eqs. (14) and (15)]. Quantities plotted in all figures are dimensionless. 


$$
\left|\lambda_{ \pm}\right|=\sqrt{\alpha_{ \pm} \beta_{ \pm}}=\left(\frac{k_{x}^{\prime}+k_{y}^{\prime}}{2}+\omega^{2} \pm \Delta\right)^{1 / 2},
$$

with $\lambda_{\mu}$ real and $b_{\mu}^{\prime}=\sqrt{\beta_{\mu} / 2 \lambda_{\mu}} Q_{\mu}^{\prime}+i \sqrt{\alpha_{\mu} / 2 \lambda_{\mu}} P_{\mu}^{\prime}$ standard bosons $\left(\left[b_{\mu}^{\prime}, b_{\nu}^{\prime \dagger}\right]=\delta_{\mu \nu}\right.$ and $\left.\left[b_{\mu}^{\prime}, b_{\nu}^{\prime}\right]=\left[b_{\mu}^{\prime \dagger}, b_{\nu}^{\prime \dagger}\right]=0\right)$ such that $H$ exhibits a discrete spectrum. In these cases the matrix representing Eq. (1) is diagonalizable with the symplectic metric, with real symplectic eigenvalues [42].

At fixed $k_{\mu}$ in Eq. (4) (charged particle in a magnetic field), Eq. (9) is valid in the following domains [42] (Fig. 1):

(A) $k_{x}>0$ and $k_{y}>0$, where $\alpha_{ \pm}>0, \beta_{ \pm}>0$, and $\lambda_{ \pm}>0$. It is the standard case of an attractive quadratic potential, where $H$ is positive definite and hence fully stable.

(B) $k_{x}<0, k_{y}<0$, and

$$
|\omega|>\omega_{c}=\frac{\sqrt{-k_{x}}+\sqrt{-k_{y}}}{2},
$$

where $\alpha_{+}>0$ and $\beta_{+}>0$ but $\alpha_{-}<0$ and $\beta_{-}<0$, implying $\lambda_{+}>0$ but $\lambda_{-}<0$ in Eq. (9). It is the case of a repulsive quadratic potential, where $H$ becomes equivalent to a standard plus an inverted oscillator if $|\omega|>\omega_{c}$ : It has no minimum energy, but is dynamically stable, as the motion remains bounded [42]. The dynamics around a quadratic potential maximum can then be stabilized by a sufficiently strong field.

(C) $k_{x}=k_{y}=0$ and $\omega \neq 0$. It is the Landau case, where $\Delta=2 \omega^{2}, \alpha_{+}=1$, and $\beta_{+}=4 \omega^{2}$, whereas $\alpha_{-}=\beta_{-}=0$, leading to $\lambda_{+}=2|\omega|$ and $\lambda_{-}=0$. Equation (9) becomes a standard plus a vanishing oscillator. Here the final choice of $b_{-}^{\prime}$ and $b_{-}^{\prime \dagger}$ is not fixed, as $\lambda_{-}=0$. We will set $b_{-}^{\prime}=\sqrt{\omega} Q_{-}^{\prime}+$ $i P_{-}^{\prime} / \sqrt{4 \omega}$, according to the $k_{\mu} \rightarrow 0$ limit of the isotropic case $k_{x}=k_{y}=k$, where $\lambda_{ \pm}=\sqrt{k^{\prime}} \pm \omega, \Delta=2 \omega \sqrt{k^{\prime}}, \gamma=\sqrt{k^{\prime}}=$ $1 / \eta, \alpha_{\mu}=\frac{1}{2} \lambda_{\mu} / \sqrt{k^{\prime}}, \beta_{\mu}=2 \lambda_{\mu} \sqrt{k^{\prime}}$, and $k^{\prime}=k+\omega^{2}$.

At fixed $k_{\mu}^{\prime}$ in Eq. (1) (rotating potential), the previous sectors are seen quite differently. Sector $(A)$ corresponds to $k_{x}^{\prime}>0, k_{y}^{\prime}>0$, and

$$
|\omega|<\omega_{c 1}^{\prime}=\min \left[\sqrt{k_{x}^{\prime}}, \sqrt{k_{y}^{\prime}}\right],
$$

indicating a maximum allowable frequency in an attractive rotating quadratic potential (right panel in Fig. 1). Sector (B) corresponds to

$$
|\omega|>\omega_{c 2}^{\prime}=\max \left[\sqrt{k_{x}^{\prime}}, \sqrt{k_{y}^{\prime}}\right]
$$

if $k_{x}^{\prime}>0$ and $k_{y}^{\prime}>0$. Thus, as the frequency $|\omega|$ is increased above $\omega_{c 1}^{\prime}$ a finite instability interval $\omega_{c 1}^{\prime}<|\omega|<\omega_{c 2}^{\prime}$ arises in the anisotropic case $k_{x}^{\prime} \neq k_{y}^{\prime}$, although dynamical stability is again recovered for $|\omega|>\omega_{c 2}^{\prime}$. In addition, sector $(B)$ also corresponds here to $k_{x}^{\prime}>0$ and $k_{y}^{\prime}<0$ (or vice versa) provided [42]

$$
\begin{aligned}
& |\omega|>\sqrt{k_{x}^{\prime}}, \quad-k_{x}^{\prime} \leqslant k_{y}^{\prime}<0 \\
& \sqrt{k_{x}^{\prime}}<|\omega|<\omega_{c 3}^{\prime}=\frac{k_{x}^{\prime}-k_{y}^{\prime}}{\sqrt{8\left(k_{x}^{\prime}+k_{y}^{\prime}\right)}}, \quad-3 k_{x}^{\prime}<k_{y}^{\prime}<-k_{x}^{\prime} .
\end{aligned}
$$

Hence a quadratic potential repulsive in one of the axes can be stabilized by increasing the frequency above $\sqrt{k_{x}^{\prime}}$ if $-3 k_{x}^{\prime}<$ $k_{y}^{\prime}<0$, although stability holds just within a finite interval if
$-3 k_{x}^{\prime}<k_{y}^{\prime}<-k_{x}^{\prime}$. Finally, the Landau case $(C)$ corresponds to $k_{x}^{\prime}=k_{y}^{\prime}=\omega^{2}$.

\section{Covariance matrix}

Both the vacuum $\left|0^{\prime}\right\rangle$ of the primed bosons $b_{\mu}^{\prime}$ in Eq. (9) and the thermal state

$$
\rho=Z^{-1} \exp [-\beta H],
$$

well defined in the stable region $(A)$ [with $\beta=1 / T>$ 0 and $\left.Z=\operatorname{Tr} \exp (-\beta H)=\sum_{\mu= \pm}\left\{2 \sinh \left(\beta \lambda_{\mu} / 2\right)\right\}^{-1}\right]$, are Gaussian states [5,7-12]. Any expectation value and in particular the entanglement between the $x$ and $y$ modes in these states will then be completely determined by the elements of the basic covariance matrix of the operators $Q_{\mu}$ and $P_{\mu}$, which we define as [12] (note that here $\left\langle Q_{\mu}\right\rangle=\left\langle P_{\mu}\right\rangle=0$ )

$$
\begin{gathered}
\mathcal{D}^{R}=\left\langle R R^{t}\right\rangle-\mathcal{M}^{R}=\left(\begin{array}{cc}
Q & L \\
L^{t} & P
\end{array}\right)-\frac{1}{2} \mathcal{M}^{R}, \\
\mathcal{M}^{R}=R R^{t}-\left(R R^{t}\right)^{t}=i\left(\begin{array}{cc}
0 & 1 \\
-1 & 0
\end{array}\right),
\end{gathered}
$$

where $R=\left(Q_{x}, Q_{y}, P_{x}, P_{y}\right)^{t}$ and hence $Q_{\mu \nu}=\left\langle Q_{\mu} Q_{\nu}\right\rangle$, $P_{\mu \nu}=\left\langle P_{\mu} P_{\nu}\right\rangle$, and $L_{\mu \nu}=\left\langle Q_{\mu} P_{\nu}+P_{\nu} Q_{\mu}\right\rangle / 2$.

Equation (17) is unitarily related to the non-negative bosonic contraction matrix [12,31]

$$
\begin{gathered}
\mathcal{D}=\left\langle Z Z^{\dagger}\right\rangle-\mathcal{M}=\left(\begin{array}{cc}
F & G \\
\bar{G} & 1+\bar{F}
\end{array}\right), \\
\mathcal{M}=Z Z^{\dagger}-\left[\left(Z^{\dagger}\right)^{t} Z^{t}\right]^{t}=\left(\begin{array}{cc}
1 & 0 \\
0 & -1
\end{array}\right),
\end{gathered}
$$

where $Z=\left(b_{x}, b_{y}, b_{x}^{\dagger}, b_{y}^{\dagger}\right)^{t}, F_{\mu \nu}=\left\langle b_{\nu}^{\dagger} b_{\mu}\right\rangle, G_{\mu \nu}=\left\langle b_{\mu} b_{\nu}\right\rangle$, and $b_{\mu}=\left(Q_{\mu}+i P_{\mu}\right) / \sqrt{2}$. Since $R=\mathcal{U} Z$, with $\mathcal{U}=\left(\begin{array}{cc}1 & 1 \\ -i & i\end{array}\right) / \sqrt{2}$, we have $\mathcal{D}^{R}=\mathcal{U} \mathcal{D U}^{\dagger}$ and $\mathcal{M}^{R}=\mathcal{U} \mathcal{M U}^{\dagger}$.

In both the vacuum and the thermal state [Eq. (16)] we have

$$
\left\langle b_{\mu}^{\prime} b_{\nu}^{\prime}\right\rangle=0,\left\langle b_{\mu}^{\prime \dagger} b_{\nu}^{\prime}\right\rangle=f_{\mu}^{\prime} \delta_{\mu \nu},
$$

where $f_{\mu}^{\prime}=0$ in the vacuum state and

$$
f_{\mu}^{\prime}=-\frac{1}{\beta} \frac{\partial \ln Z}{\partial \lambda_{\mu}}-\frac{1}{2}=\frac{1}{e^{\beta \lambda_{\mu}}-1}
$$

in the thermal state. By inverting Eq. (7), we then obtain $\left\langle Q_{x} Q_{y}\right\rangle=\left\langle P_{x} P_{y}\right\rangle=L_{\mu \mu}=0$ for $\mu=x, y$ and

$$
\begin{gathered}
\left\langle Q_{\mu}^{2}\right\rangle=\left\langle Q_{\mu}^{\prime 2}\right\rangle+\frac{\eta^{2}}{(1+\gamma \eta)^{2}}\left\langle P_{-\mu}^{\prime 2}\right\rangle, \\
\left\langle P_{\mu}^{2}\right\rangle=\frac{1}{(1+\gamma \eta)^{2}}\left\langle P_{\mu}^{\prime 2}\right\rangle+\gamma^{2}\left\langle Q_{-\mu}^{\prime 2}\right\rangle, \\
\left\langle Q_{\mu} P_{-\mu}\right\rangle=-\gamma\left\langle Q_{\mu}^{\prime 2}\right\rangle+\frac{\eta}{(1+\gamma \eta)^{2}}\left\langle P_{-\mu}^{\prime 2}\right\rangle,
\end{gathered}
$$

where

$$
\left\langle Q_{\mu}^{\prime 2}\right\rangle=\left(f_{\mu}^{\prime}+\frac{1}{2}\right) \frac{\lambda_{\mu}}{\beta_{\mu}},\left\langle P_{\mu}^{\prime 2}\right\rangle=\left(f_{\mu}^{\prime}+\frac{1}{2}\right) \frac{\lambda_{\mu}}{\alpha_{\mu}} .
$$

These averages provide all the elements of Eq. (17). The symplectic eigenvalues of $\mathcal{D}^{R}$ and $\mathcal{D}$ are coincident and given precisely by $f_{\mu}^{\prime}$ and $-1-f_{\mu}^{\prime}$ [Eqs. (21) and (22)], with physical states corresponding to $f_{\mu}^{\prime} \geqslant 0$. 
They are just the standard eigenvalues of the matrix $\mathcal{D} \mathcal{M}=\left(\begin{array}{c}F-G \\ \bar{G}-1-\bar{F}\end{array}\right) \quad$ or, equivalently, $\quad \mathcal{D}^{R} \mathcal{M}^{R}=\mathcal{U} \mathcal{D} \mathcal{M U}^{\dagger}=$ $i\left(\begin{array}{cc}-L & Q \\ -P & L^{t}\end{array}\right)-I / 2$.

\section{Vacuum entanglement}

The entanglement of the vacuum $\left|0^{\prime}\right\rangle$ is a measure of its deviation from a product state $\left|0_{x}\right\rangle \otimes\left|0_{y}\right\rangle$. It can be quantified through the entanglement entropy [13], which is just the von Neumann entropy of the reduced state $\rho_{\mu}=\operatorname{Tr}_{-\mu} \rho$ of any of the modes $(\mu=x, y)$ since for a pure state $\left(\rho=\left|0^{\prime}\right\rangle\left\langle 0^{\prime}\right|\right)$ they are isospectral. The state $\rho_{\mu}$ is a Gaussian mixed state completely determined by the reduced $2 \times 2$ covariance matrix

$$
\mathcal{D}_{\mu}^{R}=\left(\begin{array}{cc}
\left\langle Q_{\mu}^{2}\right\rangle & L_{\mu \mu} \\
L_{\mu \mu} & \left\langle P_{\mu}^{2}\right\rangle
\end{array}\right)-\frac{1}{2} \mathcal{M}^{R}
$$

whose symplectic eigenvalues are $f_{\mu}=\operatorname{det}^{1 / 2}\left[\mathcal{D}_{\mu}^{R}+\right.$ $\left.\frac{1}{2} \mathcal{M}^{R}\right]-\frac{1}{2}$ and $-1-f_{\mu}$. Here $L_{\mu \mu}=0$ and hence

$$
f_{\mu}=\sqrt{\left\langle Q_{\mu}^{2}\right\rangle\left\langle P_{\mu}^{2}\right\rangle}-\frac{1}{2}
$$

which is just the deviation of the mode uncertainty from its minimum value. The entropy of $\rho_{\mu}$ is therefore that of a boson system with average occupation $f_{\mu}$ :

$$
\begin{gathered}
S\left(\rho_{\mu}\right)=-\operatorname{Tr} \rho_{\mu} \log \rho_{\mu}=h\left(f_{\mu}\right), \\
h(f)=-f \log f+(1+f) \log (1+f),
\end{gathered}
$$

which is just a positive concave increasing function of $f_{\mu}$. The vacuum is then entangled if and only if $f_{\mu}>0$, with $S \approx$ $-f_{\mu}\left(\ln f_{\mu}-1\right)$ for $f_{\mu} \rightarrow 0$ and $S \approx \ln f_{\mu}+1$ for $f_{\mu} \rightarrow \infty$ [for base e logarithm in Eq. (30)].

In the vacuum case $\left(f_{\mu}^{\prime}=0\right)$ Eqs. (23)-(28) lead to

$$
f_{\mu}=\frac{1}{2}\left[\frac{\bar{\omega}}{\bar{\omega}_{g}}\left(\frac{\bar{\omega}_{g}^{2}+\omega^{2}}{\bar{\omega}^{2}+\omega^{2}}\right)^{1 / 2}-1\right]
$$

which is independent of $\mu$, where

$$
\bar{\omega}=\frac{\omega_{x}+\omega_{y}}{2}, \quad \bar{\omega}_{g}=\sqrt{\omega_{x} \omega_{y}}
$$

denote the arithmetic and geometric averages of the original oscillator frequencies $\omega_{\mu}=\sqrt{k_{\mu}}$. Entanglement is thus completely determined by the ratios $\omega / \bar{\omega}$ and $\bar{\omega}_{g} / \bar{\omega}$ (with $\left.\bar{\omega}_{g} / \bar{\omega} \leqslant 1\right)$ or, equivalently, $\omega / \omega_{x}$ and $\omega_{y} / \omega_{x}$. It is then nonzero for all $\omega>0$ if $\omega_{x} \neq \omega_{y}$, i.e., $\bar{\omega} \neq \bar{\omega}_{g}$ (anisotropic case). In sector $A$, the $\omega_{\mu}$ are positive, whereas in $B$ they are both imaginary, implying

$$
f_{\mu}=\frac{1}{2}\left[\frac{|\bar{\omega}|}{\left|\bar{\omega}_{g}\right|}\left(\frac{\omega^{2}-\left|\bar{\omega}_{g}\right|^{2}}{\omega^{2}-|\bar{\omega}|^{2}}\right)^{1 / 2}-1\right], \quad k_{\mu}<0 .
$$

\section{E. Thermal entanglement}

For a mixed bipartite state, such as the thermal state [Eq. (16)] at $T>0$, entanglement is a measure of its deviation from a separable state [46], i.e., from a convex combination of product states $\rho_{s}=\sum_{\alpha} q_{\alpha} \rho_{x}^{\alpha} \otimes \rho_{y}^{\alpha}$, where $q_{\alpha}>0$ and $\sum_{\alpha} q_{\alpha}=1$. Such states can be created by local operations and classical communication. For a two-mode Gaussian mixed state, entanglement can be quantified by the negativity [15], which is minus the sum of the negative eigenvalues of the partial transpose $\rho^{t_{y}}$ of the total density matrix $\rho$, measuring then the degree of violation of the positive partial transpose criterion [16,17] by the entangled state. For a two-mode Gaussian state, a positive negativity is a necessary and sufficient condition for entanglement [5].

A partial transposition with respect to $y$ implies the replacement $P_{y} \rightarrow-P_{y}$ in the full covariance matrix [Eq. (17)] [5,7-9], leading to a matrix $\tilde{\mathcal{D}}^{R}$. The negativity can then be evaluated in terms of the negative symplectic eigenvalues of this matrix, which will have eigenvalues $\tilde{f}_{\mu}$ and $-1-\tilde{f}_{\mu}$, with $\tilde{f}_{\mu} \geqslant-1 / 2$ [12]. Replacing $L_{x y}$ by $-L_{x y}$ in Eq. (17), we obtain here

$$
\begin{aligned}
\tilde{f}_{ \pm} & =\left(\tilde{\alpha} \pm \sqrt{\tilde{\alpha}^{2}-\beta^{2}}\right)^{1 / 2}-\frac{1}{2} \\
& =\sqrt{\frac{1}{2}(\tilde{\alpha}+\beta)} \pm \sqrt{\frac{1}{2}(\tilde{\alpha}-\beta)}-\frac{1}{2}
\end{aligned}
$$

where $\tilde{\alpha}$ and $\beta$ can be expressed in terms of the local symplectic eigenvalues $f_{\mu}$ [Eq. (28)] and the global symplectic eigenvalues $f_{\mu}^{\prime}$ [Eq. (22)]:

$$
\begin{aligned}
\tilde{\alpha} & =\frac{1}{2}\left(\left\langle Q_{x}^{2}\right\rangle\left\langle P_{x}^{2}\right\rangle+\left\langle Q_{y}^{2}\right\rangle\left\langle P_{y}^{2}\right\rangle\right)+\left\langle Q_{x} P_{y}\right\rangle\left\langle Q_{y} P_{x}\right\rangle \\
& =\sum_{\mu}\left[\left(f_{\mu}+\frac{1}{2}\right)^{2}-\frac{1}{2}\left(f_{\mu}^{\prime}+\frac{1}{2}\right)^{2}\right], \\
\beta & =\sqrt{\left(\left\langle Q_{x} P_{y}\right\rangle^{2}-\left\langle Q_{x}^{2}\right\rangle\left\langle P_{y}^{2}\right\rangle\right)\left(\left\langle Q_{y} P_{x}\right\rangle^{2}-\left\langle Q_{y}^{2}\right\rangle\left\langle P_{x}^{2}\right\rangle\right)} \\
& =\prod_{\mu}\left(f_{\mu}^{\prime}+\frac{1}{2}\right) .
\end{aligned}
$$

[Note that if $\tilde{\alpha}$ is replaced by $\alpha=\frac{1}{2}\left(\left\langle Q_{x}^{2}\right\rangle\left\langle P_{x}^{2}\right\rangle+\left\langle Q_{y}^{2}\right\rangle\left\langle P_{y}^{2}\right\rangle\right)-$ $\left\langle Q_{x} P_{y}\right\rangle\left\langle Q_{y} P_{x}\right\rangle$, Eq. (34) becomes $f_{ \pm}^{\prime}$.] While $f_{\mu}$ depends on $\mu$ for $T>0$, Eq. (35) depends just on the sum

$$
\begin{aligned}
\sum_{\mu}\left(f_{\mu}+\frac{1}{2}\right)^{2}= & \frac{\bar{\omega}^{2}\left(\omega^{2}+\bar{\omega}_{g}^{2}\right)}{2 \bar{\omega}_{g}^{2}\left(\omega^{2}+\bar{\omega}^{2}\right)}\left(1+2 \sum_{\mu} f_{\mu}^{\prime}\right) \\
& +\frac{\left(\omega^{2}-\bar{\omega}_{g}^{2}\right)\left(\omega^{2}+2 \bar{\omega}^{2}\right)+2 \bar{\omega}^{4}}{2\left(\omega^{2}+\bar{\omega}^{2}\right)\left(\omega^{2}+\bar{\omega}^{2}-\bar{\omega}_{g}^{2}\right)} \sum_{\mu} f_{\mu}^{\prime 2} \\
& +\frac{\omega^{2}\left[\left(\omega^{2}-\bar{\omega}_{g}^{2}\right)\left(2 \bar{\omega}^{2}-\bar{\omega}_{g}^{2}\right)+2 \bar{\omega}^{4}\right]}{\bar{\omega}_{g}^{2}\left(\omega^{2}+\bar{\omega}^{2}\right)\left(\omega^{2}+\bar{\omega}^{2}-\bar{\omega}_{g}^{2}\right)} \prod_{\mu} f_{\mu}^{\prime} .
\end{aligned}
$$

The negativity can then be expressed as [12]

$$
\begin{aligned}
N & =\frac{1}{2}\left(\operatorname{Tr}\left|\rho^{t_{y}}\right|-1\right)=\frac{1}{2}\left[\prod_{\mu} \frac{1}{1+\tilde{f}_{\mu}-\left|\tilde{f}_{\mu}\right|}-1\right] \\
& =\max \left[\frac{-\tilde{f}_{-}}{1+2 \tilde{f}_{-}}, 0\right]
\end{aligned}
$$

since only $\tilde{f}_{-}$can be negative. The entanglement condition $\tilde{f}_{-}<0$ leads to $\tilde{\alpha}>\frac{1}{8}+2 \beta^{2}$ or

$$
\sum_{\mu} f_{\mu}\left(1+f_{\mu}\right)>\sum_{\mu} f_{\mu}^{\prime}\left(1+f_{\mu}^{\prime}\right)+2 \prod_{\mu} f_{\mu}^{\prime}\left(1+f_{\mu}^{\prime}\right),
$$


which imposes a temperature-dependent lower bound on the average local occupation.

In the vacuum case $f_{\mu}^{\prime}=0$, Eq. (38) implies just $f_{\mu}>0$, while Eqs. (34)-(37) reduce to

$$
\tilde{f}_{-}=f_{\mu}-\sqrt{f_{\mu}\left(f_{\mu}+1\right)}, \quad N=f_{\mu}+\sqrt{f_{\mu}\left(f_{\mu}+1\right)},
$$

with $f_{\mu}$ given by Eq. (31), in agreement with the general results for pure Gaussian states [12]. Both $-\tilde{f}_{-}$and $N$ are again concave increasing functions of $f_{\mu}$ at $T=0$ and can be taken as alternative vacuum entanglement measures.

\section{F. Quantum discord}

Quantum discord [19] is essentially a measure of the deviation of a bipartite mixed quantum state from a classically correlated state, i.e., a state diagonal in a standard or conditional product basis. For a general bipartite system $A+B$, the quantum discord $D^{B}$ can be defined as the minimum difference between the conditional von Neumann entropy of $A$ after an unread local measurement $M_{B}$ in $B$ and the original quantum conditional entropy $S(A \mid B)=S(A, B)-S(B)[19]$ :

$$
D^{B}=\min _{M_{B}} \sum_{j} p_{j} S\left(\rho_{A / j}\right)-\left[S\left(\rho_{A B}\right)-S\left(\rho_{B}\right)\right],
$$

where, for a measurement $M_{B}$ based on local projectors $P_{j}\left(\sum_{j} P_{j}=I_{B}\right), p_{j}=\operatorname{Tr} \rho_{A B} I_{A} \otimes P_{j}$ is the probability of outcome $j$ and $\rho_{A / j}$ is the reduced state of $A$ after such an outcome. Equation (40) can be also expressed as the minimum difference between the original mutual information $I(A: B)=S(A)-S(A \mid B)$, which measures all correlations between $A$ and $B$, and that after the unread local measurement, $S(A)-\sum_{j} p_{j} S\left(\rho_{A / j}\right)$, which contains the classical part of the quantum correlations $[19,20]$.

For a pure state $\left(\rho_{A B}^{2}=\rho_{A B}\right)$ both $S\left(\rho_{A B}\right)$ and $S\left(\rho_{A / j}\right)$ vanish and $D^{B}$ reduces to the entanglement entropy $S\left(\rho_{B}\right)=$ $S\left(\rho_{A}\right)$, with $D^{A}=D^{B}$ [19]. For a mixed state, however, $D^{B}$ is not an entanglement measure, being in fact nonzero for most separable states [47] and vanishing just for those separable states of the form $\rho_{c}=\sum_{j} p_{j} \rho_{A / j} \otimes P_{j}$ (classically correlated with respect to $B$ ), which remain unaltered after the local measurement $M_{B}$. In general, $D^{B} \neq D^{A}$ for mixed states. Hence, for a bipartite system with a nondegenerate ground state in a thermal equilibrium state, such as the system under study, differences between quantum discord and entanglement and between $D^{A}$ and $D^{B}$ will arise only at finite temperature.

The exact evaluation of $D^{B}$ involves a difficult minimization over all local measurements $M_{B}$. Nonetheless, for a two-mode Gaussian state, a minimization restricted to Gaussian measurements was recently shown to be analytically feasible $[21,22,48]$. For such measurements in the present system, Eq. (40) becomes, choosing $B=y$ and using Eqs. (22) and (29)-(30),

$$
D^{y}=\min _{M_{y}} h\left(f_{x}^{M_{y}}\right)-\left[h\left(f_{+}^{\prime}\right)+h\left(f_{-}^{\prime}\right)-h\left(f_{y}\right)\right],
$$

where $f_{x}^{M_{y}}$ denotes the symplectic eigenvalue of the covariance matrix $\mathcal{D}_{x}^{M_{y}}$ associated with $\rho_{x / j}$, which depends on the $2 \times 2$ covariance matrix $\mathcal{D}_{M_{y}}$ determining the local Gaussian measurement $M_{y}$ [21,22]. The final result was provided in Ref. [22] and can be fully expressed in terms of the local invariants $A=4\left(f_{x}+\frac{1}{2}\right)^{2}, B=4\left(f_{y}+\frac{1}{2}\right)^{2}, C=2 \sum_{\mu}\left(f_{\mu}^{\prime}+\right.$ $\left.\frac{1}{2}\right)^{2}-\left(f_{\mu}+\frac{1}{2}\right)^{2}$, and $D=\prod_{\mu} 4\left(f_{\mu}^{\prime}+\frac{1}{2}\right)^{2}$, which determine the quantity $E_{\min }$ of Ref. [22], with $\min _{M_{y}} f_{x}^{M_{y}}=\frac{1}{2} \sqrt{E_{\min }}-$ $\frac{1}{2}$. It can be shown that if $D^{y}>1$ the two-mode Gaussian state is entangled [21,22]. Moreover, the only two-mode Gaussian states with $D^{y}=0$ are product states [22]. The expression for $D^{x}$ (local measurement in $\left.x\right)$ is obviously similar $(x \leftrightarrow y$ in previous formulas).

\section{RESULTS}

\section{A. Vacuum entanglement}

Let us now analyze the main features of Eq. (31). We first consider fixed $k_{\mu}$ in Eq. (4) (charged particle in a magnetic field). In the isotropic case $\omega_{x}=\omega_{y}, \bar{\omega}=\bar{\omega}_{g}$, and $f_{\mu}=0$ $\forall \omega$. There is no entanglement since $L_{z}$ commutes in this case with $H$ and leaves the isotropic product vacuum invariant. For $\left|\omega_{x}-\omega_{y}\right| \ll \bar{\omega}$, Eq. (31) leads to

$$
f_{\mu} \approx \frac{\omega^{2}}{16 \bar{\omega}^{2}\left(\bar{\omega}^{2}+\omega^{2}\right)}\left(\omega_{x}-\omega_{y}\right)^{2}+O\left(\left(\omega_{x}-\omega_{y}\right)^{4}\right),
$$

indicating a quadratic vanishing of $f_{\mu}$ in this limit. Entanglement also vanishes for $\omega \rightarrow 0$ (no coupling), where

$$
f_{\mu} \approx \frac{1}{4}\left(\frac{1}{\bar{\omega}_{g}^{2}}-\frac{1}{\bar{\omega}^{2}}\right) \omega^{2}+O\left(\omega^{4}\right) .
$$

In contrast, for $\omega \rightarrow \infty$, a remarkable feature is that $f_{\mu}$ approaches a finite limit, which depends just on the anisotropy $\omega_{y} / \omega_{x}$ : For $\omega \gg|\bar{\omega}|$ Eq. (31) leads to

$$
f_{\mu} \approx \frac{1}{2}\left[\frac{\bar{\omega}}{\bar{\omega}_{g}}-1\right]+O\left(\omega^{-2}\right) .
$$

In sector $A, f_{\mu}$, and hence $S\left(\rho_{\mu}\right)$, is an increasing function of $\omega$ (Fig. 2). Mode entanglement is then enhanced just by increasing the field, although it will saturate for strong fields. This saturation is a consequence of the balance between the oscillator part and the coupling $\omega L_{z}$ in Eq. (1), as $k_{\mu}^{\prime}=$ $k_{\mu}+\omega^{2}$ also becomes large, thus reducing $\left\langle Q_{\mu}^{2}\right\rangle$ : For $\omega \rightarrow \infty$, $\left\langle Q_{\mu}^{2}\right\rangle \approx \frac{\bar{\omega}}{2 \omega_{\mu}} \omega^{-1} \rightarrow 0$ while $\left\langle P_{\mu}^{2}\right\rangle \approx \frac{\bar{\omega}}{2 \omega_{-\mu}} \omega \rightarrow \infty$, leading to the finite limit [Eq. (44)].

In contrast, $f_{\mu}$, and hence entanglement, will diverge at the edges of the dynamically stable region. For instance, if $\omega_{y} \rightarrow$ $0, \bar{\omega} \rightarrow \omega_{x} / 2$ whereas $\bar{\omega}_{g} \rightarrow 0$, implying $f_{\mu} \propto 1 / \sqrt{\omega_{y}}$ :

$$
f_{\mu} \approx \frac{1}{2}\left[\left(\frac{\bar{\omega}}{2 \omega_{y}}\right)^{1 / 2} \frac{\omega}{\sqrt{\bar{\omega}^{2}+\omega^{2}}}-1\right]
$$

and hence $S\left(\rho_{\mu}\right) \approx \frac{1}{2} \log \left(\omega_{x} / \omega_{y}\right)$ plus constant terms. This divergence stems from that of $\left\langle Q_{y}^{2}\right\rangle$ (or $\left\langle P_{x}^{2}\right\rangle$ ) in this limit, with $\left\langle P_{y}^{2}\right\rangle$ and $\left\langle Q_{x}^{2}\right\rangle$ remaining constant [Eqs. (23)-(26)].

In the repulsive sector $B, f_{\mu}$ diverges for $\omega \rightarrow|\bar{\omega}|=\omega_{c}$ [Eq. (11)], where both $\left\langle Q_{\mu}^{2}\right\rangle$ and $\left\langle P_{\mu}^{2}\right\rangle$ diverge:

$$
f_{\mu} \approx \frac{1}{2}\left[\left(\frac{|\bar{\omega}|}{\omega-|\bar{\omega}|}\right)^{1 / 2}\left(\frac{|\bar{\omega}|^{2}-\left|\bar{\omega}_{g}\right|^{2}}{2\left|\bar{\omega}_{g}\right|^{2}}\right)^{1 / 2}-1\right] .
$$



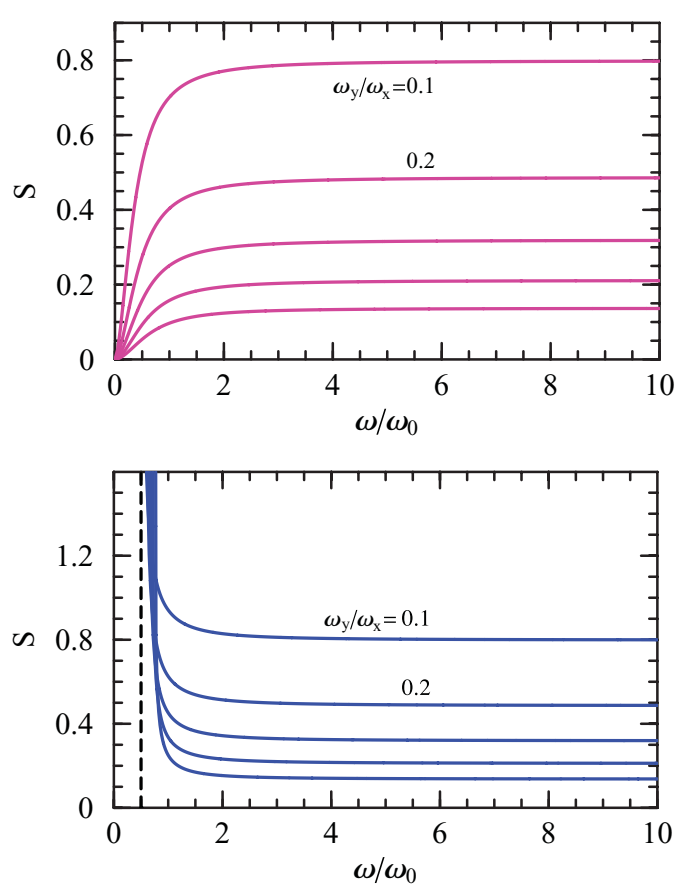

FIG. 2. (Color online) Entanglement entropy $S=S\left(\rho_{\mu}\right)$ [Eq. (29)] between the two modes as a function of $\omega$ in the vacuum of the Hamiltonian [Eq. (1)] at fixed $k_{\mu}=\omega_{\mu}^{2}$ for ratios $\omega_{y} / \omega_{x}=$ $0.1, \ldots, 0.5$ and $\omega_{0}=\left|\omega_{x}\right|$. The top panel corresponds to sector $A$ (attractive potential $k_{\mu}>0$ ) and the bottom panel corresponds to sector $B$ (repulsive potential $k_{\mu}<0$ and $|\omega|>\omega_{c}$ ), where $S$ diverges for $\omega \rightarrow \omega_{c}$ [Eq. (11)]. In both cases $S$ approaches the same finite limit for $\omega \rightarrow \infty$.

It is then seen that here $f_{\mu}$, and hence $S\left(\rho_{\mu}\right)$, decreases as $\omega$ increases from $\omega_{c}$ (Fig. 2, bottom panel), i.e., as the system becomes dynamically stabilized by the field, reaching, for $\omega \rightarrow \infty$, the same previous limit [Eq. (44)]. At fixed $\left|k_{y} / k_{x}\right|$, the vacuum entanglement is then strictly larger in the unstable sector $B\left(k_{\mu}<0\right)$.

At fixed $k_{\mu}^{\prime}$ (rotating potential) the behavior with frequency is quite different (Fig. 3). We should now replace

$$
\omega_{\mu}=\sqrt{\omega_{\mu}^{\prime 2}-\omega^{2}}, \quad \omega_{\mu}^{\prime}=\sqrt{k_{\mu}^{\prime}}
$$

in Eqs. (31) and (32). For $\omega_{x}^{\prime}=\omega_{y}^{\prime}$ there is of course no entanglement. For $\left|\omega_{x}^{\prime}-\omega_{y}^{\prime}\right| \ll \omega_{x}$, we have

$$
f_{\mu} \approx \frac{\omega^{2}}{16\left(\omega^{2}-\omega_{x}^{\prime 2}\right)^{2}}\left(\omega_{x}^{\prime}-\omega_{y}^{\prime}\right)^{2}+O\left(\left(\omega_{x}^{\prime}-\omega_{y}^{\prime}\right)^{4}\right) .
$$

Entanglement also vanishes for $\omega \rightarrow 0$, where Eq. (43) still holds $\left(\omega_{\mu}^{\prime}=\omega_{\mu}\right.$ at $\left.\omega=0\right)$.

Conversely, as $\omega$ increases, $f_{\mu}$ increases rapidly and, in contrast with the previous case, diverges for $\omega \rightarrow \omega_{c 1}^{\prime}$ [Eq. (12)], where, assuming $\omega_{c 1}^{\prime}=\omega_{y}^{\prime}<\omega_{x}^{\prime}$,

$$
f_{\mu} \approx \frac{1}{2}\left[\left(\frac{\omega_{y}^{\prime 3}\left(\omega_{x}^{\prime 2}-\omega_{y}^{\prime 2}\right)}{2\left(\omega_{y}^{\prime}-\omega\right)\left(3 \omega_{y}^{\prime 2}+\omega_{x}^{\prime 2}\right)^{2}}\right)^{1 / 4}-1\right],
$$

implying $S\left(\rho_{\mu}\right) \approx \frac{1}{4} \ln \left[\omega_{y}^{\prime} /\left(\omega_{y}^{\prime}-\omega\right)\right]$ plus constant terms. In this limit $\left\langle Q_{y}^{2}\right\rangle$ and $\left\langle P_{x}^{2}\right\rangle$ diverge while $\left\langle Q_{x}^{2}\right\rangle$ and $\left\langle P_{y}^{2}\right\rangle$ stay constant, as $\omega_{y}=\sqrt{\omega_{y}^{\prime 2}-\omega^{2}} \rightarrow 0$. As $\omega$ increases further,
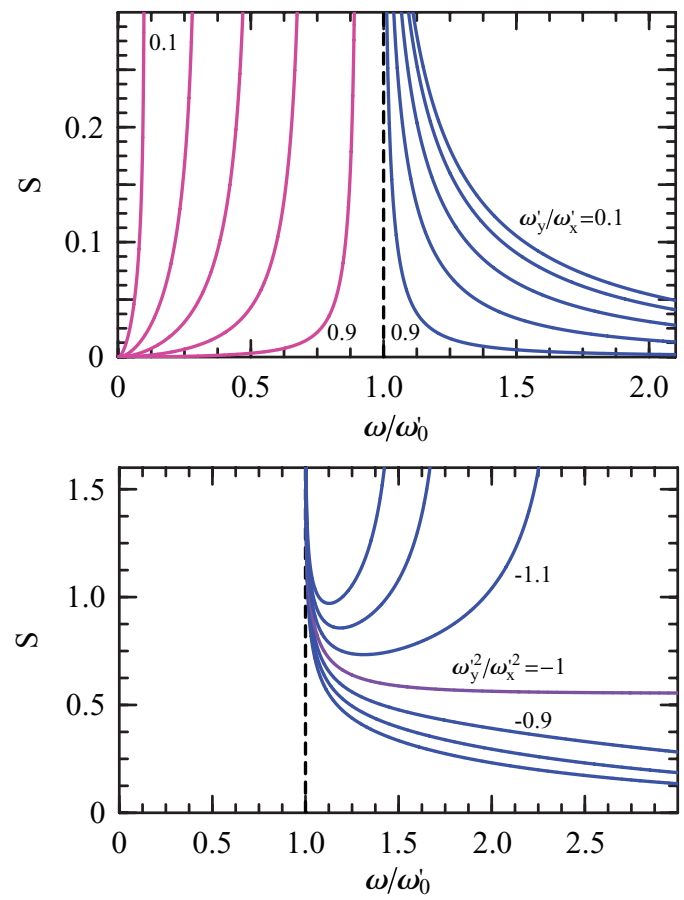

FIG. 3. (Color online) Entanglement entropy between the two modes as a function of $\omega$ in the vacuum of the Hamiltonian [Eq. (1)] at fixed $k_{\mu}^{\prime}=\omega_{\mu}^{\prime 2}$ for ratios $\omega_{y}^{\prime} / \omega_{x}^{\prime}=0.1,0.3, \ldots, 0.9$ (top) and $\left(\omega_{y}^{\prime} / \omega_{x}^{\prime}\right)^{2}=-1.3,-1.2, \ldots,-0.7$ (bottom), with $\omega_{0}^{\prime}=\omega_{x}^{\prime}$. The top panel corresponds to $k_{\mu}^{\prime}>0$, with the positive-definite sector $A$ on the left, where $S$ diverges for $\omega \rightarrow \omega_{c 1}^{\prime}=\omega_{y}^{\prime}$, and the nonpositive sector $B_{1}$ on the right $\left(\omega>\omega_{c 2}^{\prime}=\omega_{x}^{\prime}\right)$, where $S$ diverges at $\omega_{x}^{\prime}$ and vanishes for $\omega \rightarrow \infty$. The bottom panel corresponds to $k_{x}^{\prime}>0$ and $k_{y}^{\prime}<0$ (sector $B_{2}$ ), where $S$ diverges for $\omega \rightarrow \omega_{x}^{\prime}$ and also $\omega \rightarrow \omega_{c 3}^{\prime}$ [Eq. (15)] if $\left(\omega_{y}^{\prime} / \omega_{x}^{\prime}\right)^{2}<-1$. In the critical case $\left(\omega_{y}^{\prime} / \omega_{x}^{\prime}\right)^{2}=-1$ $\left(k_{y}^{\prime}=-k_{x}^{\prime}\right), f_{\mu}$ and $S$ saturate for large $\omega$ [Eq. (52)].

the system enters the instability window, although for $\omega>\omega_{c 2}^{\prime}$ [Eq. (13)], it recovers a discrete spectrum, entering sector $B_{1}$. For $\omega \rightarrow \omega_{c 2}^{\prime}, f_{\mu}$ diverges as in Eq. (49), with $\omega_{y}^{\prime} \leftrightarrow \omega_{x}^{\prime}$ if $\omega_{c 2}^{\prime}=\omega_{x}^{\prime}$.

In sector $B_{1}, f_{\mu}$, and hence the entanglement, decreases as $\omega$ increases, vanishing for $\omega \rightarrow \infty$, in contrast with the behavior at fixed $k_{\mu}$ in sector $A$. In this limit the vacuum of $H$ now becomes that associated with $\omega L_{z}$, which is an isotropic product Gaussian state with $L_{z}=0$ and hence zero entanglement. Then $\left\langle Q_{\mu}^{2}\right\rangle$ and $\left\langle P_{\mu}^{2}\right\rangle$ stay finite and their product approaches minimum uncertainty, leading to

$$
f_{\mu} \approx \frac{\left(\omega_{x}^{\prime 2}-\omega_{y}^{\prime 2}\right)^{2}}{32 \omega^{2}\left(\omega_{x}^{\prime 2}+\omega_{y}^{\prime 2}\right)}+O\left(\omega^{-4}\right) .
$$

In the unstable domain $B_{2}$, the behavior with $\omega$ is the same as in $B_{1}$ when $k_{x}^{\prime}>0$ and $-k_{x}^{\prime}<k_{y}^{\prime}<0$. However, for $k_{x}^{\prime}>$ 0 and $-3 k_{x}^{\prime}<k_{y}^{\prime}<-k_{x}^{\prime}$, we also have the upper instability limit in Eq. (15) $\left(\omega_{c 3}^{\prime}\right)$. In this case $f_{\mu}$ first decreases with increasing $\omega$, reaching a minimum, but then starts again to increase, diverging for $\omega \rightarrow \omega_{c 3}^{\prime}$ where now both $\left\langle Q_{\mu}^{2}\right\rangle$ and $\left\langle P_{\mu}^{2}\right\rangle$ diverge, leading to

$$
f_{\mu} \approx \frac{1}{2}\left[\left(\frac{\omega_{c 3}^{\prime}}{2\left(\omega_{c 3}^{\prime}-\omega\right)}\right)^{1 / 2}-1\right] .
$$




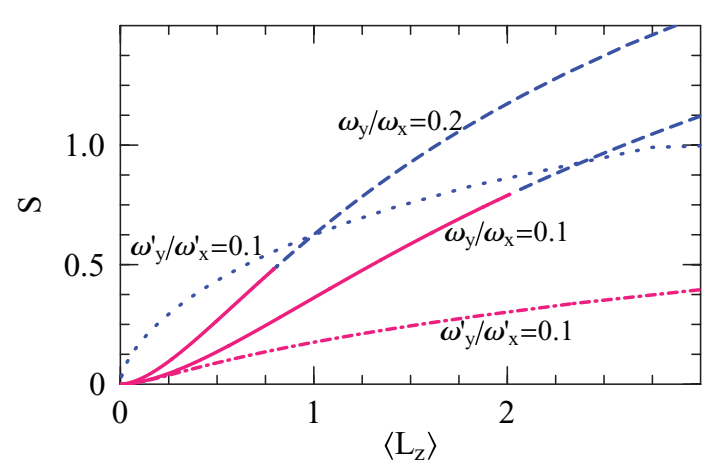

FIG. 4. (Color online) Entanglement entropy between the two modes as a function of the scaled average angular momentum $\left\langle L_{z}\right\rangle=$ $\left\langle\mathcal{L}_{z}\right\rangle / \hbar$ [Eq. (5)] in the vacuum of Eq. (1) at fixed $k_{\mu}=\omega_{\mu}^{2}$ (solid and dashed lines) and at fixed $k_{\mu}^{\prime}=\omega_{\mu}^{\prime 2}$ (dash-dotted and dotted lines) for selected ratios $\omega_{y} / \omega_{x}$ and $\omega_{y}^{\prime} / \omega_{x}^{\prime}$ and variable $\omega$. The solid and dash-dotted lines (red) correspond to sector $A$ ( $k_{\mu}>0$ or $k_{\mu}^{\prime}>0$ and $\left.|\omega|<\omega_{y}^{\prime}\right)$, the dashed and dotted lines (blue) correspond to sectors $B\left(k_{\mu}<0\right.$ and $\left.|\omega|>\omega_{c}\right)$ and $B_{1}\left(k_{\mu}^{\prime}>0\right.$ and $\left.|\omega|>\omega_{x}^{\prime}\right) . S$ is an increasing function of $\left|\left\langle L_{z}\right\rangle\right|$ in all sectors.

We then obtain different $O\left(\omega-\omega_{c}\right)^{-1 / 4}$ and $O\left(\omega_{c}-\omega\right)^{-1 / 2}$ divergences of $f_{\mu}$ at the stability borders $\omega_{x}^{\prime}$ and $\omega_{c 3}^{\prime}$, respectively.

In the special critical case $k_{y}^{\prime}=-k_{x}^{\prime}\left(\omega_{y}^{\prime}=i \omega_{x}^{\prime}\right)$, where $\omega_{c 3}^{\prime} \rightarrow \infty$, Eq. (31) leads to

$$
f_{\mu}=\frac{1}{2}\left[\left(1+\frac{\omega^{2}}{\sqrt{\omega^{4}-\omega_{x}^{\prime 2}}}\right)^{1 / 2}-1\right]
$$

and hence to a finite asymptotic limit $f_{\mu}=\frac{1}{2}(\sqrt{2}-1)$ for $\omega \rightarrow \infty$, in contrast with Eq. (50), as also appreciated in Fig. 3. In this limit $\left\langle Q_{\mu}^{2}\right\rangle$ diverges whereas $\left\langle P_{\mu}^{2}\right\rangle$ vanishes, the product approaching $1 / 2$. Hence, as $\omega$ increases, $f_{\mu}$ vanishes if $k_{y}^{\prime}<-k_{x}^{\prime}$, saturates if $k_{y}^{\prime}=-k_{x}^{\prime}$, and diverges (at $\omega=\omega_{c 3}^{\prime}$ ) if $-3 k_{x}^{\prime}<k_{y}^{\prime}<-k_{x}^{\prime}$.

The behavior of $f_{\mu}$, and hence $S\left(\rho_{\mu}\right)$, with $\omega$ is qualitatively similar to that of the average angular momentum $\left\langle L_{z}\right\rangle$. At fixed $k_{\mu}$, the latter also saturates for $\omega \rightarrow \infty\left[\left\langle L_{z}\right\rangle \rightarrow\left(\bar{\omega} / \bar{\omega}_{g}\right)^{2}-1\right]$ and diverges for $\omega \rightarrow \omega_{c}\left[\left\langle L_{z}\right\rangle \propto\left(\omega-\omega_{c}\right)^{-1 / 2}\right]$, whereas at fixed $k_{\mu}^{\prime}$ it diverges for $\omega \rightarrow \omega_{c i}^{\prime}\left[\left\langle L_{z}\right\rangle \propto\left(\omega-\omega_{c i}^{\prime}\right)^{-1 / 2}\right.$ for $i=1,2]$ and vanishes for $\omega \rightarrow \infty$. Entanglement is then an increasing function of $\left|\left\langle L_{z}\right\rangle\right|$ at fixed $k_{\mu}$ or $k_{\mu}^{\prime}$, as seen in Fig. 4, although it is not fully determined by $\left|\left\langle L_{z}\right\rangle\right|$, as the latter is not invariant under local transformations (in contrast with $\left.f_{\mu}\right)$. At fixed $\left\langle L_{z}\right\rangle$, higher ratios $k_{y} / k_{x}<1$ originate a higher entanglement (Fig. 4). For small $\omega,\left\langle L_{z}\right\rangle \propto \omega$ and hence $f_{\mu} \propto$ $\left\langle L_{z}\right\rangle^{2}$ for small $\left\langle L_{z}\right\rangle$ in sector $A$. However, at fixed $k_{\mu}^{\prime},\left\langle L_{z}\right\rangle$ also vanishes for large $\omega$, where $\left\langle L_{z}\right\rangle \propto \omega^{-3}$. Hence, in sector $B_{1}$ and according to Eq. (50), $f_{\mu} \propto\left\langle L_{z}\right\rangle^{2 / 3}$ for small $\left\langle L_{z}\right\rangle$, leading to an infinite initial slope (dotted line in Fig. 4). At fixed $\left\langle L_{z}\right\rangle$ and $k_{\mu}^{\prime}$, entanglement is then stronger in the unstable sector $B_{1}$ $\left(\omega>\omega_{c 2}^{\prime}\right)$. An exceptional behavior occurs in the critical case $k_{y}^{\prime}=-k_{x}^{\prime}$ [Eq. (52)], where for $\omega \rightarrow \infty,\left\langle L_{z}\right\rangle \propto \omega^{-2}$ vanishes while $f_{\mu}$ remains finite. In this special limit there is finite entanglement with vanishing angular momentum. In contrast, close to the divergences, $f_{\mu} \propto\left\langle L_{z}\right\rangle\left(\omega \rightarrow \omega_{c}\right)$ or $\left\langle L_{z}\right\rangle^{1 / 2}(\omega \rightarrow$ $\left.\omega_{c i}^{\prime}, i=1,2\right)$, implying $S\left(\rho_{\mu}\right) \propto \ln \left\langle L_{z}\right\rangle$ for large $\left\langle L_{z}\right\rangle$.
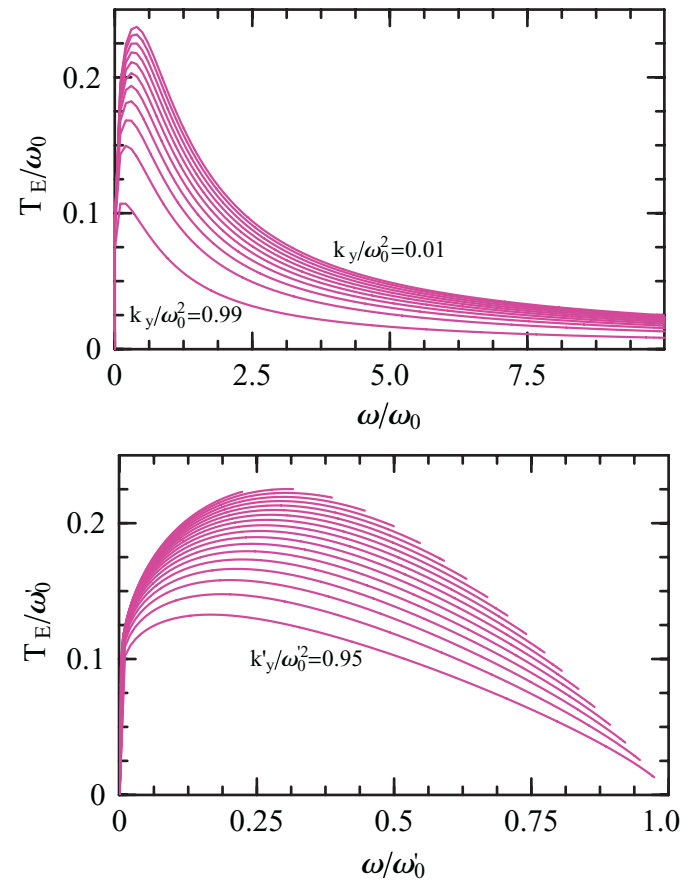

FIG. 5. (Color online) Scaled limit temperatures for entanglement $T_{E}$ at fixed $k_{\mu}$ (top) and at fixed $k_{\mu}^{\prime}$ (bottom), as a function of frequency for different ratios $k_{y} / k_{x}=0.01,0.1, \ldots, 0.9,0.99$ (top) and $k_{y}^{\prime} / k_{x}^{\prime}=$ $0.05,0.1, \ldots, 0.95$ (bottom), with $\omega_{0}=\omega_{x}=\sqrt{k_{x}}$ and $\omega_{0}^{\prime}=\omega_{x}^{\prime}=$ $\sqrt{k_{x}^{\prime}}$. At fixed $k_{\mu}, T_{E}$ vanishes for large $\omega$ [Eq. (55)] whereas at fixed $k_{\mu}^{\prime}$, it approaches a finite value at the upper stability limit $\omega_{c 1}^{\prime}=\sqrt{k_{y}^{\prime}}$.

\section{B. Thermal entanglement}

Let us now examine the thermal entanglement in the stable sector $A$. We first depict in Fig. 5 the limit temperature for entanglement $T_{E}$, determined from the condition $\tilde{f}_{-}=0$ [equality in Eq. (38)]. This temperature remains finite for all values of $k_{\mu}$ or $k_{\mu}^{\prime}$, including the edge of the sector $\left(k_{\mu} \rightarrow 0\right.$ or $|\omega| \rightarrow \sqrt{k_{\mu}^{\prime}}$ ), where the vacuum $f_{\mu}$ diverges. At the edge, $\lambda_{-} \rightarrow 0$ and hence a finite $T$ already gives rise to a spread over all energy levels $\left(f_{-}^{\prime} \rightarrow \infty\right)$, which diminishes and eventually eliminates the entanglement. A related fundamental effect is that at finite $T>0$, entanglement does not diverge at the edge, but stays finite or vanishes, depending on the value of $T$.

More precisely, for $\omega_{y} \rightarrow 0$ and fixed $\omega_{x}>0, \lambda_{+} \rightarrow$ $\sqrt{4 \omega^{2}+\omega_{x}^{2}}$ whereas $\lambda_{-} \approx \omega_{y} \omega_{x} / \lambda_{+}$, implying $f_{-}^{\prime} \approx T / \lambda_{-} \approx$ $T \lambda_{+} / \omega_{x} \omega_{y}$. Hence, in this limit Eqs. (34)-(36) lead to

$$
\tilde{f}_{-}=\frac{1}{2}\left[\left(\frac{T \lambda_{+}^{4}\left(1+2 f_{+}^{\prime}\right)^{2}}{\omega_{x}^{2}\left[2 \omega^{2} \lambda_{+}\left(1+2 f_{+}^{\prime}\right)+T \omega_{x}^{2}\right]}\right)^{1 / 2}-1\right]
$$

which remains finite and above $-1 / 2$ if $T>0$. This implies a finite negativity in this limit if $T>0$, with $N \propto T^{-1 / 2}$ for $T \rightarrow 0$ according to Eq. (37). Therefore, at finite temperature the vacuum divergences of the entanglement can only be probed indirectly through the $T^{-1 / 2}$ behavior of $N$ near the edge at sufficiently low $T$. 
In addition, Eq. (53) entails a finite limit temperature $T_{E}$, obtained from the condition $\tilde{f}_{-}=0$ in Eq. (53):

$$
T_{E}=\frac{2\left(1+2 f_{+}^{\prime}\right) \omega^{2} \omega_{x}^{2} \lambda_{+}}{\left(1+2 f_{+}^{\prime}\right)^{2} \lambda_{+}^{4}-\omega_{x}^{4}},
$$

which is a transcendental equation for $T_{E}\left(f_{+}^{\prime}\right.$ depends on $\left.T_{E}\right)$. The maximum limit temperature $T_{E}^{M}$ at fixed $k_{x}=\omega_{x}^{2}$ or $T_{E}^{\prime M}$ at fixed $k_{x}^{\prime}=\omega_{x}^{\prime 2}$ is in fact obtained in this limit $\left(\omega_{y}=0\right.$ or $\left.\omega_{y}^{\prime}=\omega\right)$ : At fixed $k_{x}, T_{E}^{M} \approx 0.24 \omega_{x}$, attained at $\omega \approx 0.38 \omega_{x}$, while at fixed $k_{x}^{\prime}, T_{E}^{\prime M} \approx 0.23 \omega_{x}^{\prime}$, attained at $\omega \approx 0.28 \omega_{x}^{\prime}$.

At fixed $k_{\mu}$, the limit temperature $T_{E}$ as a function of $\omega$ exhibits first a maximum and then vanishes for $\omega \rightarrow \infty$ (top panel in Fig. 5), i.e., in the limit where the vacuum entanglement saturates. The reason is that $\lambda_{-}$also vanishes for $\omega \rightarrow \infty\left(\lambda_{-} \approx \omega_{x} \omega_{y} / 2 \omega\right)$, implying $T_{E} \propto \omega^{-1}$ in this limit:

$$
T_{E} \approx \frac{\omega_{x} \omega_{y}}{2 \omega \ln \frac{\omega_{x}+\omega_{y}}{\omega_{x}-\omega_{y}}} .
$$

In fact, for $\omega \rightarrow \infty$ and fixed $k_{\mu}$ (with $k_{y}<k_{x}$ ), Eqs. (34)-(36) lead to

$$
\tilde{f}_{-} \approx \frac{1}{2}\left[\left(\frac{\left(1+2 f_{-}^{\prime}\right) \omega_{y}}{\omega_{x}}\right)^{1 / 2}-1\right],
$$

such that $\tilde{f}_{-}=0$ for $1+2 f_{-}^{\prime}=\omega_{x} / \omega_{y}$, which leads to Eq. (55). In contrast, for $\omega \rightarrow 0, T$ vanishes logarithmically $\left(T_{E} \propto-1 / \ln \omega\right)$ and the same occurs for $\omega_{y} \rightarrow \omega_{x}$ since in these limits $\lambda_{ \pm}$remain both finite whereas the negativity vanishes. At fixed $T$, we then obtain a finite-frequency window for entanglement, which narrows for increasing temperature or decreasing anisotropy, as seen in Fig. 5 and also Fig. 6, where the negativity [Eq. (37)] is depicted. Entanglement ceases to be correlated with $\left\langle L_{z}\right\rangle$ as the temperature increases $\left(\left\langle L_{z}\right\rangle \propto \omega T\right.$ for high $T$ ).

At fixed $k_{\mu}^{\prime}$, the behavior of $T_{E}$ and $N$ look quite different, as now $\omega$ is bounded above by $\omega_{y}^{\prime}$ (assuming $\omega_{y}^{\prime}<\omega_{x}^{\prime}$ ). For $\omega \rightarrow$ $\omega_{y}^{\prime}, T_{E}$ is then determined by Eq. (54), with $\omega_{x} \rightarrow \sqrt{\omega_{x}^{\prime 2}-\omega^{2}}$, and remains finite. Actually, as verified in the bottom panel of Fig. 5, $T_{E}$ acquires in this border its maximum value as $\omega$ increases at fixed $k_{\mu}^{\prime}$ if $\omega_{y}^{\prime}<\omega_{y c}^{\prime} \approx 0.28 \omega_{x}^{\prime}$, while if $\omega_{y}^{\prime}>$ $\omega_{y c}^{\prime}$ the maximum is attained at an intermediate frequency. Consequently, at fixed $T<T_{E}^{\prime M}$ there is again entanglement within a certain frequency window, which extends up to the stability border $\omega=\omega_{y}^{\prime}$ if $\omega_{y}^{\prime}<\omega_{y c}^{\prime}$ or $T<T_{E}$ at $\omega=\omega_{y}^{\prime}$ (bottom panel in Fig. 6). The absolute maximum $T_{E}^{\prime M}$ is obtained at this border precisely at $\omega_{y}^{\prime}=\omega_{y c}^{\prime}$. For $\omega \rightarrow 0$ or $\omega_{y}^{\prime} \rightarrow \omega_{x}^{\prime}, T_{E}$ again decreases logarithmically.

\section{Comparison with quantum discord}

We finally compare in Fig. 7 the thermal behavior of the negativity with that of the Gaussian quantum discord $D^{y}$ [Eq. (41)] and also $D^{x}$. For reference we have also plotted the entropy of one of the modes $(x)$, no longer a measure of entanglement, just to indicate its coincidence with both $D^{y}$ and $D^{x}$ for $T \rightarrow 0$. While at $T=0$ the negativity is just an increasing function of the entanglement entropy [Eqs. (29)-(39)], and hence of the quantum discord, the behavior for $T>0$ is quite different. Although exhibiting a
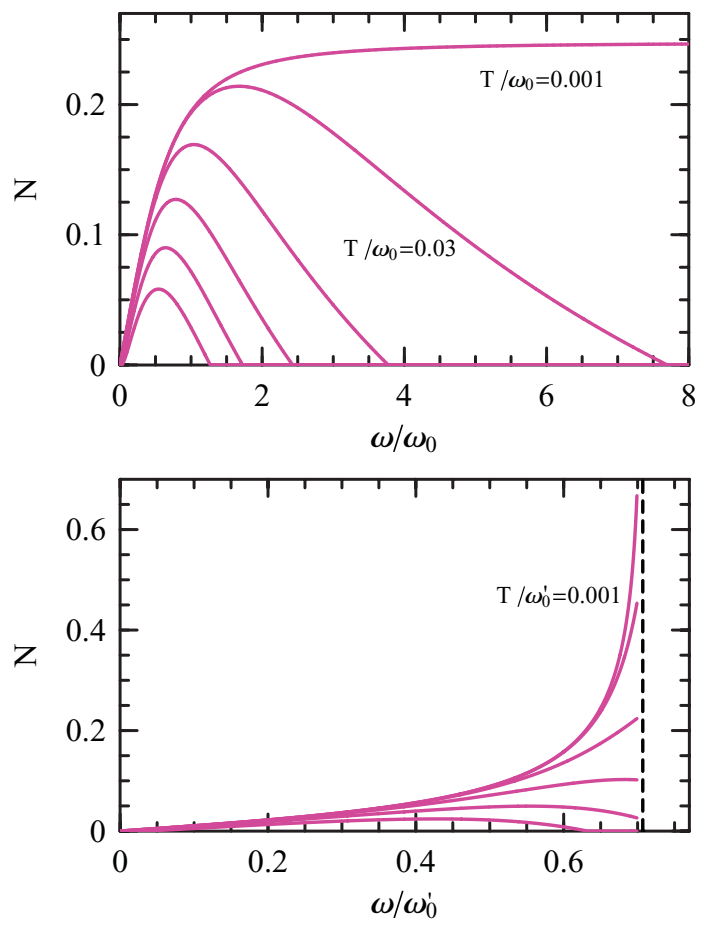

FIG. 6. (Color online) Negativity as a function of frequency at increasing temperatures $T / \omega_{0}=0.001,0.03,0.06, \ldots, 0.15$, at fixed $k_{\mu}$ (top), for $k_{y} / k_{x}=0.2$, and at fixed $k_{\mu}^{\prime}$ (bottom), for $k_{y}^{\prime} / k_{x}^{\prime}=0.5$. At $T>0, N$ is finite and nonzero just within a finite-frequency window.

similar initial decreasing trend [essentially due to the initial increase of the total entropy $S\left(\rho_{A B}\right)$ in Eq. (40)], the Gaussian discord then starts to increase [due to the increase in the first term of Eq. (40)], vanishing only asymptotically for $T \rightarrow \infty$. Such a revival of the discord with increasing $T$ was also observed in spin systems $[49,50]$ and reflects the presence of quantum correlations in the excited eigenstates, which at these temperatures lead to a separable yet not classically correlated (in the sense of Sec. II F) thermal state. Since $D^{\mu}>1$ implies entanglement [21,22], one can ensure here that $D^{\mu}<1$ after the vanishing of the negativity $\left(T>T_{E}\right)$, although this may not prevent $D^{\mu}$ from reaching a higher value than at $T=0$ at some

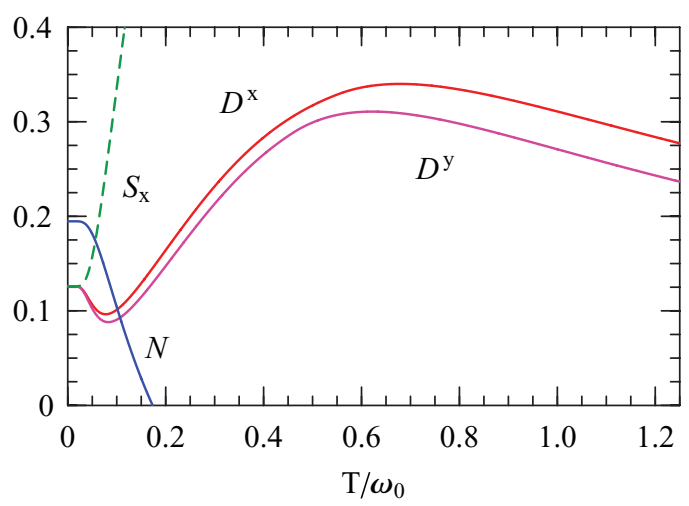

FIG. 7. (Color online) Negativity $N$, single-mode entropy $S_{x}=$ $S\left(\rho_{x}\right)$, and the quantum discords $D^{y}$ and $D^{x}$ as a function of temperature for $\omega=\omega_{0}=\sqrt{k_{x}}$ and $k_{y}=0.2 k_{x}$. 
intermediate temperature, as seen in Fig. 7. For $T \rightarrow \infty$ we actually obtain, from Eq. (41) and the expression of Ref. [22], that $D^{\mu} \propto T^{-1}$ :

$$
D^{y} \approx \frac{\omega^{2}}{2 T \sqrt{\omega^{2}+\omega_{x}^{2}}},
$$

with a similar expression for $D^{x}$ after replacing $\omega_{x}$ by $\omega_{y}$. Hence, for high $T, D^{\mu}$ becomes independent of $\omega_{\mu}$, with $D^{x}>$ $D^{y}$ asymptotically if $\omega_{y}<\omega_{x}$, as verified in Fig. 7. We also note that the discord remains finite for $T>0$ in the whole sector $A$.

\section{CONCLUSION}

We have analyzed the entanglement induced by an angular momentum coupling of two harmonic modes. Full analytic expressions for the vacuum entanglement entropy and the thermal negativity were derived. The model exhibits a rich phase structure and admits distinct physical realizations (a particle in a magnetic field in an anisotropic harmonic trap or a particle in a rotating harmonic trap), which lead to different entanglement behaviors with the relevant control parameter. For instance, in sector $A$ (stable vacuum), entanglement saturates for strong fields in the first case, but diverges at a finite frequency in the second case. Vacuum entanglement diverges at the onset of instabilities, being correlated with the average angular momentum and reaching higher values in unstable domains dynamically stabilized by the field or rotation. In contrast, thermal entanglement is finite and nonzero just below a finite limit temperature within a reduced-frequency window, diverging only for $T \rightarrow 0$ at the instability borders. We have also shown that after a short initial common trend, the thermal behavior of the Gaussian quantum discord becomes substantially different from that of entanglement, vanishing only asymptotically. It would be interesting to verify that a similar thermal behavior is also exhibited by other related measures of quantum correlations [48,51].

\section{ACKNOWLEDGMENTS}

The authors acknowledge support from CONICET (L.R.) and CIC (R.R.) of Argentina.
[1] M. A. Nielsen and I. L. Chuang, Quantum Computation and Quantum Information (Cambridge University Press, Cambridge, 2000).

[2] V. Vedral, Introduction to Quantum Information Science (Oxford University Press, Oxford, 2006).

[3] L. Amico, R. Fazio, A. Osterloh, and V. Vedral, Rev. Mod. Phys. 80, 516 (2008).

[4] R. Horodecki, P. Horodecki, M. Horodecki, and K. Horodecki, Rev. Mod. Phys. 81, 865 (2009).

[5] R. Simon, Phys. Rev. Lett. 84, 2726 (2000).

[6] L. M. Duan, G. Giedke, J. I. Cirac, and P. Zoller, Phys. Rev. Lett. 84, 2722 (2000).

[7] R. F. Werner and M. M. Wolf, Phys. Rev. Lett. 86, 3658 (2001).

[8] K. Audenaert, J. Eisert, M. B. Plenio, and R. F. Werner, Phys. Rev. A 66, 042327 (2002).

[9] G. Adesso, A. Serafini, and F. Illuminati, Phys. Rev. A 70, 022318 (2004); A. Serafini, G. Adesso, and F. Illuminati, ibid. 71, 032349 (2005).

[10] M. Cramer, J. Eisert, M. B. Plenio, and J. Dreißig, Phys. Rev. A 73, 012309 (2006).

[11] G. Adesso and F. Illuminati, Phys. Rev. A 78, 042310 (2008).

[12] J. M. Matera, R. Rossignoli, and N. Canosa, Phys. Rev. A 82, 052332 (2010)

[13] C. H. Bennett, H. J. Bernstein, S. Popescu, and B. Schumacher, Phys. Rev. A 53, 2046 (1996).

[14] K. Zyczkowski, P. Horodecki, A. Sanpera, and M. Lewenstein, Phys. Rev. A 58, 883 (1998).

[15] G. Vidal and R. F. Werner, Phys. Rev. A 65, 032314 (2002).

[16] A. Peres, Phys. Rev. Lett. 77, 1413 (1996).

[17] M. Horodecki, P. Horodecki, and R. Horodecki, Phys. Lett. A 223, 1 (1996).

[18] S. L. Braunstein and P. van Loock, Rev. Mod. Phys. 77, 513 (2005).
[19] H. Ollivier and W. H. Zurek, Phys. Rev. Lett. 88, 017901 (2001).

[20] L. Henderson and V. Vedral, J. Phys. A 34, 6899 (2001).

[21] P. Giorda and M. G. A. Paris, Phys. Rev. Lett. 105, 020503 (2010)

[22] G. Adesso and A. Datta, Phys. Rev. Lett. 105, 030501 (2010).

[23] A. Datta, A. Shaji, and C. M. Caves, Phys. Rev. Lett. 100, 050502 (2008).

[24] E. Knill and R. Laflamme, Phys. Rev. Lett. 81, 5672 (1998).

[25] A. Datta, S. T. Flammia, and C. M. Caves, Phys. Rev. A 72, 042316 (2005).

[26] D. Cavalcanti, L. Aolita, S. Boixo, K. Modi, M. Piani, and A. Winter, Phys. Rev. A 83, 032324 (2011).

[27] A. Streltsov, H. Kampermann, and D. Bruß, Phys. Rev. Lett. 106, 160401 (2011).

[28] M. Piani, S. Gharibian, G. Adesso, J. Calsamiglia, P. Horodecki, and A. Winter, Phys. Rev. Lett. 106, 220403 (2011).

[29] J. G. Valatin, Proc. R. Soc. London 238, 132 (1956).

[30] A. Feldman and A. H. Kahn, Phys. Rev. B 1, 4584 (1970).

[31] P. Ring and P. Schuck, The Nuclear Many-Body Problem (Springer, New York, 1980).

[32] J. P. Blaizot and G. Ripka, Quantum Theory of Finite Systems (MIT Press, Cambridge, MA, 1986).

[33] A. V. Madhav and T. Chakraborty, Phys. Rev. B 49, 8163 (1994).

[34] M. Linn, M. Niemeyer, and A. L. Fetter, Phys. Rev. A 64, 023602 (2001).

[35] M. Ö. Oktel, Phys. Rev. A 69, 023618 (2004).

[36] A. L. Fetter, Phys. Rev. A 75, 013620 (2007).

[37] A. Aftalion, X. Blanc, and N. Lerner, Phys. Rev. A 79, 011603(R) (2009).

[38] A. Aftalion, X. Blanc, and J. Dalibard, Phys. Rev. A 71, 023611 (2005); S. Stock et al., Laser Phys. Lett. 2, 275 (2005).

[39] I. Bloch, J. Dalibard, and W. Zwerger, Rev. Mod. Phys. 80, 885 (2008).

[40] A. L. Fetter, Rev. Mod. Phys. 81, 647 (2009). 
[41] J. Pěrina, Z. Hradil, and B. Jurčo, Quantum Optics and Fundamentals of Physics (Kluwer, Dordrecht, 1994); N. Korolkova, J. Pěrina, Opt. Commun. 136, 135 (1996).

[42] R. Rossignoli and A. M. Kowalski, Phys. Rev. A 79, 062103 (2009).

[43] A. P. Hines, R. H. McKenzie, and G. J. Milburn, Phys. Rev. A 67, 013609 (2003).

[44] H. T. Ng and P. T. Leung, Phys. Rev. A 71, 013601 (2005).

[45] A. V. Chizhov and R. G. Nazmitdinov, Phys. Rev. A 78, 064302 (2008).
[46] R. F. Werner, Phys. Rev. A 40, 4277 (1989).

[47] A. Ferraro, L. Aolita, D. Cavalcanti, F. M. Cucchietti, and A. Acin, Phys. Rev. A 81, 052318 (2010).

[48] L. Mišta, R. Tatham, D. Girolami, N. Korolkova, and G. Adesso, Phys. Rev. A 83, 042325 (2011).

[49] T. Werlang and G. Rigolin, Phys. Rev. A 81, 044101 (2010).

[50] J. Maziero, H. C. Guzman, L. C. Celeri, M. S. Sarandy, and R. M. Serra, Phys. Rev. A 82, 012106 (2010).

[51] R. Rossignoli, N. Canosa, and L. Ciliberti, Phys. Rev. A 82, 052342 (2010). 